\title{
Endothelial dysfunction and the role of hypertension in Nepalese subjects with major coronary risk factors
}

\author{
Mani Prasad Gautam, ${ }^{1}$ Samir Gautam, ${ }^{2}$ Usha Ghimire, ${ }^{3}$ Sogunuru Guruprasad, ${ }^{4}$ Rabin Bhattacharya, ${ }^{4}$ \\ Gangapatnam Subramanyam ${ }^{4}$
}

${ }^{1}$ Bharatpur Hospital, Bharatpur, Nepal

${ }^{2}$ Gautam Buddha Community Heart Hospital, Butwal, Nepal,

${ }^{3}$ Communication and Management Institute Private Limited, Kathmandu, Nepal

${ }^{4}$ Department of Cardiology, College of Medical Sciences, Bharatpur, Nepal

Corresponding Author: Mani Prasad Gautam, Bharatpur Hospital, Bharatpur, Nepal, Email: manigautam@gmail.com

\begin{abstract}
Background and Aims: Hypertension is one of the major coronary heart disease risk factors. Endothelial dysfunction is thought to be the preclinical vascular changes in the pathogenesis of atherosclerosis and it its major manifestation; coronary heart disease. This study was designed to assess the endothelial function in subjects with major coronary risk factors including hypertension.

Methods: The study was conducted in a tertiary referral centre in Central Nepal. A cross-sectional study was conducted on the cohort of 100 subjects with at least one out of six major coronary heart disease risk factors. JNC 7 criteria were used to define hypertension. Brachial artery flow-mediated dilation was measured using high-resolution ultrasound. Endothelial dysfunction was defined as flow-mediated dilation value $<9.99 \%$. The association between endothelial dysfunction and hypertension was assessed by univariate and multivariate analysis.

Results: Out of 100 subjects with coronary risk factors (mean age $46.75 \pm 9.95$ years, mean number of risk factors $2.81 \pm 1.17$ ), $65 \%$ subjects were hypertensive and $35 \%$ were non-hypertensive. Hypertension as a risk factor was evident in $80.39 \%$ and $48.98 \%$ subjects with abnormal and normal endothelial response respectively $(\mathrm{p}=0.001)$. In addition, $63.08 \%$ and $28.57 \%$ subjects with and without hypertension had endothelial dysfunction respectively $(\mathrm{p}=0.01)$. Hypertension was the only coronary risk factor associated with endothelial dysfunction in a multivariate model $(\mathrm{p}=0.04)$.

Conclusion: Hypertension was strongly associated with endothelial dysfunction and it could be the best predictor of endothelial dysfunction and subsequent coronary heart disease in coronary heart diseases risk factor cohorts.
\end{abstract}

Key words: Hypertension, endothelial dysfunction, coronary risk factors

\section{Introduction}

Hypertension is the most common condition predisposing to coronary heart disease. The National Cholesterol Education Program's Adult Treatment Panel III (ATP III) report identified the hypertension as a risk factor for cardiovascular disease. ${ }^{1}$ It is readily identifiable reversible risk factor and its burden is rising and projected to affect one third of the world's population by the year 2025. Currently, high blood pressure (BP) causes about 54\% of stroke and $47 \%$ of coronary heart disease (CHD) worldwide. ${ }^{2}$

Endothelial dysfunction (ED) is thought to be the preclinical vascular changes in the pathogenesis of atherosclerosis including systemic hypertension and coronary heart disease. Alteration in endothelial function and impaired balance between endothelial relaxing and constricting factors play an important role in the pathogenesis of atherosclerosis. ${ }^{3}$ ED is both an early marker of vascular disease and a precursor in the development of atherosclerosis. ${ }^{4,5}$ Studies have shown that the presence of endothelial dysfunction identifies individuals at increased risk for cardiovascular disease events. $6,7,8,9$
Coronary artery disease is associated with endothelial dysfunction which can be diagnosed preclinically. ${ }^{10} \mathrm{ED}$, defined by a lower flow-mediated dilation (FMD) value, has been found to be associated with cardiovascular events. ${ }^{11}$ The brachial-artery flow-mediated dilation (FMD) has been extensively used to identify the ED proposed as a surrogate marker of cardiovascular disease. ${ }^{12,13,14}$ Studies have reported diverse association between HTN and ED. Brunner et al and others reported the presence of endothelial dysfunction in hypertensive subjects. ${ }^{15,16,17}$ In contrast to this, Cockcroft et al had reported normal endothelial function in hypertensive subjects. ${ }^{18}$

This study was designed to assess the endothelial function in subjects with various coronary risk factors including hypertension.

\section{Methods}

The study was conducted in College of Medical Sciences Teaching Hospital, Bharatpur, a tertiary referral centre. A cross- 
sectional study was designed. A cohort of 100 subjects with at least one out of six major CHD risk factors: hypertension, diabetes, obesity, age more than 45 years in male and more than 55 years in female, dyslipidemia and smoking were selected. The subjects with major coronary risk factors without obvious coronary heart disease and definitive structural and functional heart diseases were included in the study cohort. JNC 7 criteria were used to define hypertension - any blood pressure level on treatment for hypertension and the blood pressure more or equal to $140 / 90$ $\mathrm{mmHg}$ in two separate readings taken at least 1 week apart in those without treatment. ${ }^{19}$ Other CHD risk factors were defined as per the Adult Treatment Panel - III; ${ }^{1}$ diabetes was defined as the subjects on treatment for diabetes or fasting plasma glucose more or equal to $126 \mathrm{mg} / \mathrm{dl}$, or postprandial plasma glucose level more or equal to $200 \mathrm{mg} / \mathrm{dl}$ or random blood sugar level more or equal to $200 \mathrm{mg} / \mathrm{dl}$ in the presence of symptoms suggestive of raised blood sugar level. Similarly dyslipidemia was defined as any one of the following - LDL more than $130 \mathrm{mg} / \mathrm{dl}$ or HDL less than $40 \mathrm{mg} / \mathrm{dl} .{ }^{1}$ Any subjects with age 20 to 70 years, having at least one major CHD risk factor mentioned above and clinically stable were included in the cohort. Subjects with coronary heart disease, terminal illness, any other active illness and regularly taking medicines having effect on endothelial function such as statins, ACE inhibitors were excluded from the study. The study was approved by Institutional Review Board. An informed consent was taken from each patient and the required information was recorded as per predefined performa.

Each subject had undergone detail clinical evaluation followed by basic and advanced biochemical and haematological laboratory tests including renal, liver and thyroid function test and lipid profile. In addition, these subjects were also undergone non-invasive comprehensive cardiac evaluation including electrocardiography and echocardiography to rule out coronary heart disease and advanced cardiac conditions. Brachial artery flow-mediated dilation was measured using high-resolution ultrasound as described elsewhere..$^{20,21}$ After a 15-min resting period in a air-conditioned room $\left(22^{\circ} \mathrm{C}\right.$ to $\left.25^{\circ} \mathrm{C}\right)$, the FMD was assessed in the subject's right arm in the recumbent position by ACUSON 300, Premium Edition (SIEMENS, Germany) with a 10.1 MHz linear array transducer. The brachial artery was imaged and the diameter at end diastole was measured. Three readings of brachial artery diameter were taken and mean was calculated which was the baseline diameter. A pressure cuff was placed on the forearm distal to the target artery and it was inflated to at least $50 \mathrm{~mm} \mathrm{Hg}$ above systolic pressure until no blood flow was detected through the brachial artery with the Doppler probe for 5 minutes. After $5 \mathrm{~min}$, the cuff was released, and the diameter of the brachial artery was measured at 30,60, 90 and 120 seconds. The second reading i.e. 60 seconds measurement was taken to calculate the change in brachial artery diameter and it was expressed in percentages.

The study was analysed in a case-control fashion. The cohort was divided in two group based on the presence of hypertension. The cases included subjects with hypertension and remaining subjects were taken as controls and comparison was made between them. Endothelial dysfunction was defined as flow-mediated dilation value $<9.99 \% .{ }^{22,23}$ Based on the presence of endothelial dysfunction the subjects were also divided into two groupswithandwithoutEDandvarious parameterswere compared between them. The various confounding factors in hypertensive and non-hypertensive groups were analysed. In addition, similar comparison was also made between subjects with and without ED.

Data were analysed by using SPSS 17.0. The categorical data were expressed in percentages and analyzed using $\chi^{2}$ test. Continuous data were presented in the form of mean with standard deviation and the significance of difference between two means was tested by using student $t$ test. The association between endothelial dysfunction and hypertension was assessed by univariate and multivariate analysis.

\section{Results}

One hundred subjects with various coronary risk factors were selected and studied (Figure 1). Mean age was $46.75 \pm 9.95$ years. The mean number of coronary risk factors on these subjects was $2.81 \pm 1.17$. Majority of the subjects $(65 \%)$ were hypertensive. The proportion of subjects with other risk factors was: DM - $11 \%$, Smoking - 19\%, Age (Men > 45, Women > 55) - 44\%, BMI High risk $(>27.5 \mathrm{Kg} / \mathrm{m} 2)-66 \%$ and Dyslipidemia $-81 \%$ (Table 1).

Hypertension as a risk factor was evident in $80.39 \%$ and $48.98 \%$ subjects with abnormal and normal endothelial response respectively $(p=0.001)$. In addition, $63.08 \%$ and $28.57 \%$ subjects with and without hypertension had endothelial dysfunction respectively $(\mathrm{p}=0.01)$.

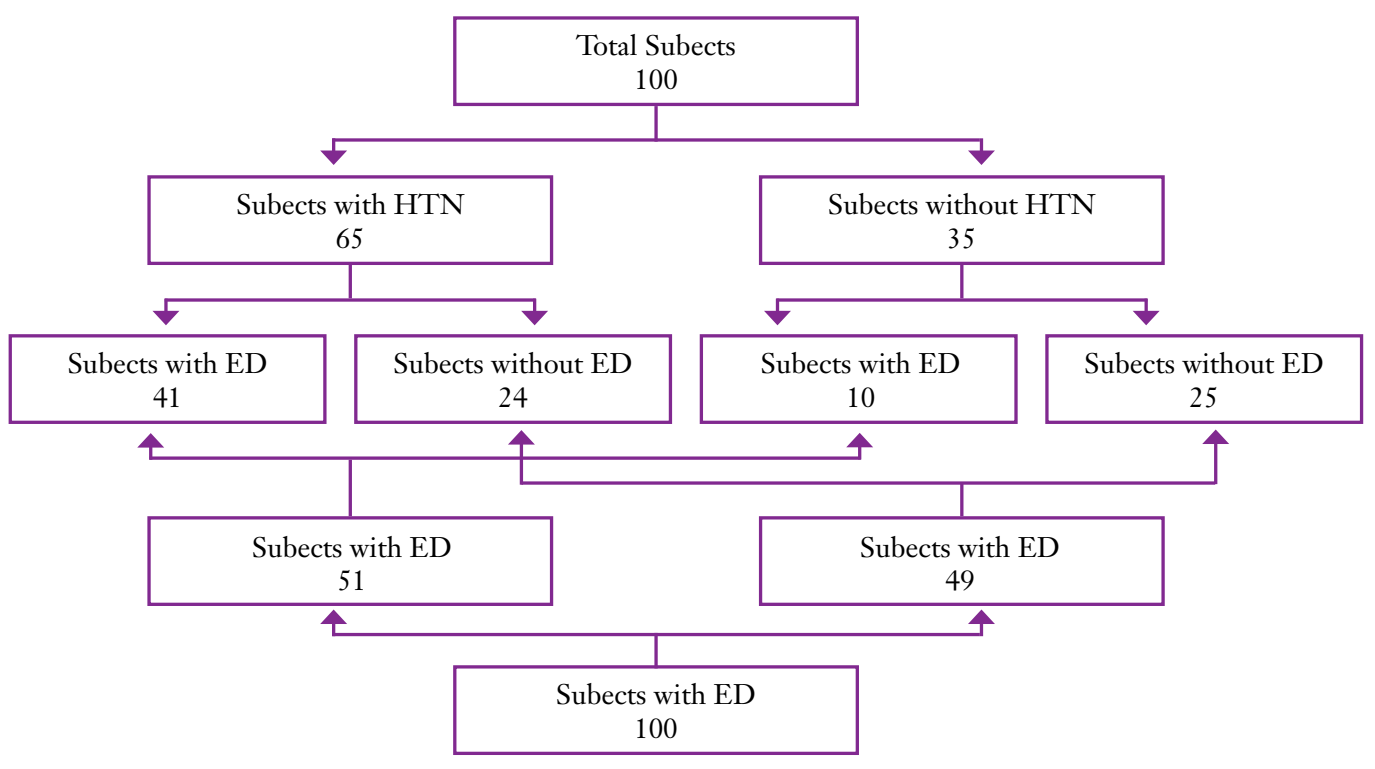

Fig. 1. Study subjects. HTN, Hypertension, ED, Endothelial dysfunction 
Table 1. Studied parameters in subjects with and without hypertension (HTN)

\begin{tabular}{llll} 
Parameters & $\begin{array}{l}\text { Subjects with HTN } \\
\mathbf{N = 6 5}\end{array}$ & $\begin{array}{l}\text { Subjects without HTN } \\
\mathbf{N}=35\end{array}$ & P value \\
\hline Age (Years) & $49.8 \pm 8.85$ & $41.09 \pm 9.47$ & $<0.001$ \\
\hline Male & $33(50.77)$ & $9(25.71)$ & 0.01 \\
Change in BAD $(\mathrm{mm})$ & $0.34 \pm 0.19$ & $0.43 \pm 0.16$ & 0.02 \\
\hline FMD & $8.77 \pm 4.75$ & $12.1 \pm 4.3$ & 0.01 \\
\hline Endothelial dysfunction $(\%)$ & $41(63.08)$ & $10(28.57)$ & 0.01 \\
\hline Number of CHD risk factors & $3.23 \pm 1.15$ & $2.06 \pm 0.68$ & $<0.001$
\end{tabular}

Table 2. Endothelial function parameters in subjects with and without endothelial dysfunction.

\begin{tabular}{llll} 
Parameters & $\begin{array}{l}\text { Subjects with ED } \\
\mathbf{N}=\mathbf{5 1}\end{array}$ & $\begin{array}{l}\text { Subjects without ED } \\
\mathbf{N}=49\end{array}$ & p Value \\
\hline Mean Age (yrs) & $50.57 \pm 98.94$ & $42.78 \pm 9.44$ & $<0.001$ \\
\hline Male $(\%)$ & $27(52.94)$ & $15(30.61)$ & 0.02 \\
\hline Mean increase in BAD $(\mathrm{mm})$ & $0.23 \pm 0.13$ & $0.51 \pm 0.08$ & $\mathrm{NA}$ \\
\hline Mean FMD & $5.85 \pm 2.75$ & $14.06 \pm 1.94$ & $\mathrm{NA}$ \\
\hline Mean number of risk factors & $3.43 \pm 1.04$ & $2.18 \pm 0.86$ & $<0.001$ \\
\hline Number of CHD risk factors & $3.23 \pm 1.15$ & $2.06 \pm 0.68$ & $<0.001$
\end{tabular}

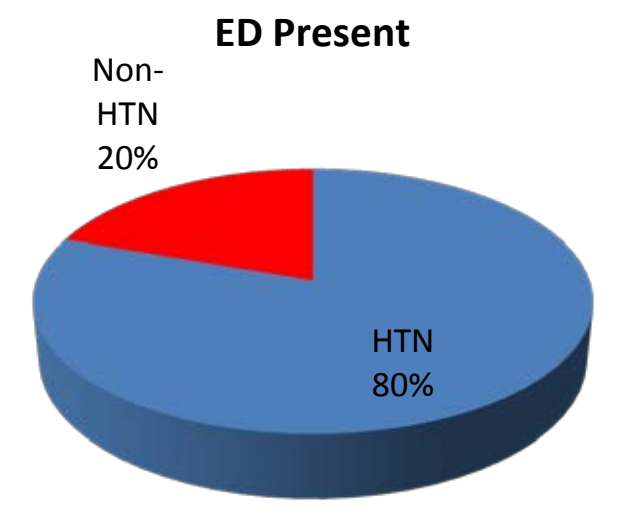

\section{ED Absent}

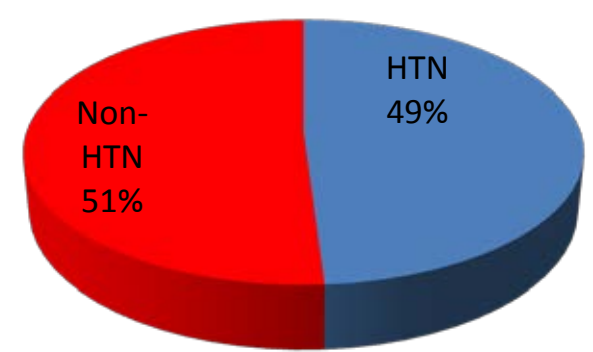

Figure 2. Endothelial function (ED) in subjects with and without hypertension (HIN)

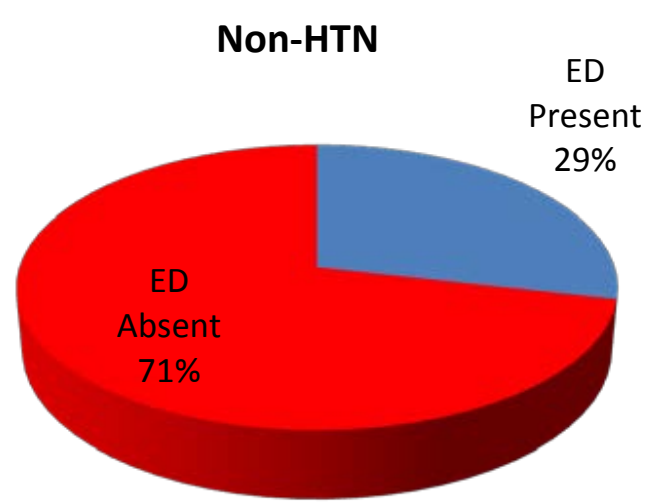

ED

Absent

HTN

$37 \%$

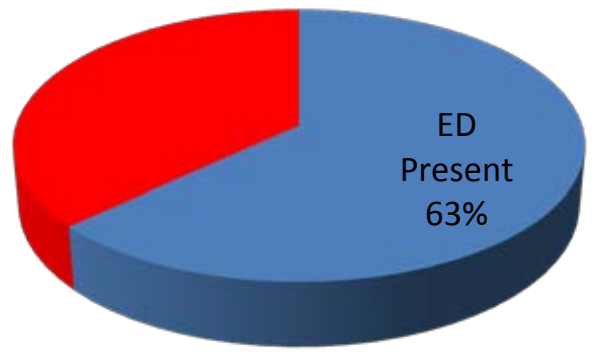

Figure 3. Hypertension in subjects with and without endothelial dysfunction 
Table 3: Univariate association between risk factors and endothelial dysfunction defined as FMD $\leq 9.99 \%$ in study subjects

\begin{tabular}{|llll} 
& OR & CI (95\%) & P value \\
\hline Age (yrs) & 1.09 & $1.044-1.149$ & $<0.001$ \\
\hline Smoking & 0.3 & $0.099-0.912$ & 0.03 \\
\hline Dyslipidemia & 4.05 & $1.34-12.25$ & 0.01 \\
\hline Diabetes & 5.7 & $1.18-27.6$ & 0.03 \\
\hline Hypertension & 4.27 & $1.35-10.39$ & 0.001 \\
\hline BMI of High Risk & 1.16 & $0.52-2.59$ & 0.71
\end{tabular}

\begin{tabular}{llll}
\multicolumn{2}{l}{ Table 4: Multivariate association between risk factors and endothelial dysfunction defined as FMD $\leq 9.99 \%$ in study subjects. } \\
\hline HTN & OR & CI (95\%) & P value \\
\hline DM & 6.436 & $1.067-38.809$ & 0.042 \\
Smoking & 11.463 & $0.272-483.962$ & 0.202 \\
\hline Sex & 1.127 & $0.056-22.517$ & 0.938 \\
Dyslipidemia & 1.749 & $0.284-10.759$ & 0.547 \\
\hline Age & 2.905 & $0.277-30.483$ & 0.374 \\
\hline
\end{tabular}

In a subanalysis where all subjects were divided in tertiles according to FMD values, the rate of hypertension was falling from $87.88 \%$ to $63.64 \%$ to $44.12 \%$ in subjects in lower, middle and upper tertiles respectively. Hypertension was associated with ED in univariate analysis (OR 4.27, CI 1.35-10.39 p = 0.001). Other CHD risk factors such as diabetes, smoking, age, and dyslipidemia were also associated with $\mathrm{ED}$ in univariate analysis $(\mathrm{p}<0.05)$. But high risk BMI including obesity and abdominal obesity were not associated with ED even in univariate analysis ( $p=0.71$ and 0.68$)$. In multivariate analysis where the role of various confounding factors was considered, hypertension was the only CHD risk factor associated with ED (OR 6.436, CI $1.067-38.809, \mathrm{p}=0.042)$. Association of ED with other risk factors was not statistically significant.

\section{Discussion}

Various studies have shown that both the hypertension and endothelial dysfunction are risk factors and provide additive prognostic values in predicting cardiovascular events. ${ }^{11}$ In our study, the rate of endothelial dysfunction was $63.08 \%$ and $28.57 \%$ in hypertensive and non-hypertensive subjects respectively $(\mathrm{p}=$ $0.01)$. Similarly, the rate of hypertension was $80.39 \%$ and $48.98 \%$ in subjects with and without endothelial dysfunction $(\mathrm{p}=0.001)$. Brunner $\mathrm{H}$ et $\mathrm{al},{ }^{15}$ Benjamin EJ et $\mathrm{al},{ }^{16}$ and Felmeden DC et $\mathrm{al}^{17}$ also has reported similar association between HTN and ED in their study. There are reports showing the predictive power of endothelial dysfunction for the development of hypertension in future. Rossi et al. had suggested that the presence of endothelial dysfunction can increase the susceptibility to develop hypertension and diabetes. ${ }^{24}$ Similar association between ED and HTN was also seen in the Framingham cohort analysis of 2883 individuals ( $53 \%$ female) using FMD as a measure of endothelial function. ${ }^{16}$
In our study, the rate of endothelial dysfunction was $63.08 \%$ and $28.57 \%$ in hypertensive and non-hypertensive subjects respectively $(\mathrm{OR}=4.2, \mathrm{p}=0.01)$. Similarly, the rate of hypertension was $80.39 \%$ and subjects were divided in tertiles according to FMD values, the rate of hypertension was falling from $87.88 \%$ to $63.64 \%$ to $44.12 \%$ in subjects in lower, middle and upper tertiles respectively. It showed strong relationship between hypertension and endothelial dysfunction. Moreover, the hypertension as a CHD risk factor was the only factor having strong power as a predictor of ED. It was significantly associated with ED in univariate (OR 4.27, CI 1.35-10.39 $\mathrm{p}=0.001)$ and multivariate analysis (OR 6.436, CI 1.067-38.809, $\mathrm{p}=0.042)$. Similar findings of the presence of endothelial dysfunction in hypertensive subjects was also reported by Brunner et al. ${ }^{15}$ and others. ${ }^{16,17}$ Benjamin et $\mathrm{a}^{16}$ examined a measure of endothelial function, brachial artery flow-mediated dilation (FMD), expressed as both percent (FMD\%) and actual dilation by ultrasound with the occlusion cuff below the elbow in 2883 Framingham Study participants (52.9\% women; mean age, 61 years). They concluded that the increasing age, systolic blood pressure, BMI, and smoking were associated with lower FMD\% in the community-based sample, whereas prior exercise and increasing heart rate were associated with higher FMD\%. Thus the Framingham cohort analysis has clearly demonstrated a relationship with blood pressure and other CHD risk factors. ${ }^{16}$ Felmeden et $\mathrm{al}^{17}$ had studied 84 hypertensive patients ( 74 men; mean age, 64 years; SD 8 ) by calculating an LDL score, with higher scores being equivalent to a greater proportion of the more atherogenic LDL subfractions. High-resolution ultrasound was used to assess endotheliumdependent brachial artery FMD after reactive hyperemia after vessel occlusion. Baseline levels were compared with 61 age- and gender-matched healthy normotensive control subjects. Mean LDL score was higher and FMD impaired in hypertensive subjects compared with control subjects. 
In contrast to this, Cockcroft et al. ${ }^{18}$ had reported normal endothelial function in hypertensive subjects. They had reassessed this issue by measuring forearm blood flow by plethysmography during the infusion of vasodilators into the brachial arteries of 95 subjects: 37 normotensive controls and 58 patients with essential hypertension. They recorded that the vasodilator responses to nitroprusside, acetylcholine, carbachol, and isoproterenol were also similar in separate groups of normotensive controls and hypertensive subjects, whether the subjects had never been treated for hypertension or had had therapy withheld for two weeks. In contrast to previous studies, their findings suggest that selective impairment of the responsiveness of the forearm vasculature to muscarinic agonists is not universal in patients with essential hypertension.

However, there are reports showing not only endothelial dysfunction in hypertensive subjects but also the predictive power of endothelial dysfunction for the development of hypertension in future. Rossi et al..$^{25}$ had suggested that the presence of endothelial dysfunction can increase the susceptibility to develop hypertension and thus hypertension can be seen not only as a collateral feature of established risk factors, but also a possible pathogenetic mechanism for their onset. Chang et al conducted a study in subjects having exerciseinduced hypertension to clarify whether this condition is related to endothelial dysfunction or not by evaluating the endothelial function of the brachial artery with high-resolution ultrasound technique, ${ }^{25}$ and measuring the concentration of NO2/NO3 and cyclic guanosine monophosphate (GMP). ${ }^{26}$ They concluded that patients with exercise-induced hypertension have impaired endothelium-dependent vasodilatation and the endothelial dysfunction may play an important role in exercise-induced hypertension. They also proposed that the patients with exercise-induced hypertension have poor endothelial dependent vasodilatation due to an impaired nitric oxide /cyclic GMP pathway and this might play a significant role in increasing blood pressure during exercise with inadequate peripheral adjustment to changing cardiac output.

In overall, despite some contrast reports regarding the association of hypertension and endothelial dysfunction, majorities are in the favour of the deleterious effects of hypertension on endothelial function and our findings are also in accordance with these observations. Further study with large sample size and design to assess causal relationship between hypertension and endothelial dysfunction could be more informative and can further clarify the issue.

This Study was designed to assess the association between ED and HTN; therefore, a causal relationship cannot be drawn. All the subjects had at least one (up to six) major CHD risk factors and comparison was made with the subjects having other CHD risk factors, therefore the effect of other risk factors in endothelial function might have interference in the analysis. Another important limitation in this study was the bias caused by cardiology OPD based enrollment of the study subjects. This could be the reason for the higher proportion of hypertensive subjects in the study cohort. Moreover, in the logistic regression models although final outcome with the multivariate analysis does show a significant Odds with the hypertensive for endothelial dysfunction and a significant $\mathrm{P}$ value, this only signified a statistical significance and not a very strong clinical significance due to a wide ranging confidence interval (CI). This must be noted while interpreting the study and drawing a conclusion. Similarly, the small sample size and cross-sectional nature of the study with its inherent limitations should be taken in consideration while generalizing these findings.

\section{Conclusions}

Among the studied coronary risk factors, hypertension, diabetes, smoking, age, dyslipidemia were associated with endothelial dysfunction - a surrogate marker, precursor or risk factor of CHD as shown in univariate analysis. The study was unable to establish the association of other coronary risk factors and endothelial dysfunction. Among these coronary risk factors only the hypertension was strongly associated as shown in univariate as well as in multivariate analysis, with endothelial dysfunction and could be the strong and best predictor of endothelial dysfunction and subsequent coronary heart disease. But the wide ranging confidence interval in this association should be taken in consideration while interpreting the present study and needs on further research with appropriate design and methodology.

\section{References}

1. Third Report of the National Cholesterol Education Program (NCEP) Expert Panel on Detection, Evaluation, and Treatment of High Blood Cholesterol in Adults (Adult Treatment Panel III) final report. Circulation 2002;106:3143-421.

2. Lawes CM, Vander Hoorn S, Rodgers A. Global burden of blood-pressure-related disease, 2001. Lancet 2008; 371:1513-8.

3. Ross R. The pathogenesis of atherosclerosis: a perspective for the 1990s. Nature 1993;362(6423):801-9.

4. Dzau VJ. Theodore Cooper Lecture: Tissue angiotensin and pathobiology of vascular disease: a unifying hypothesis. Hypertension 2001;37(4):1047-52.

5. Ross R. Atherosclerosis--an inflammatory disease. N Engl J Med 1999;340(2):115-26.

6. Halcox JP, Schenke WH, Zalos G, et al. Prognostic value of coronary vascular endothelial dysfunction. Circulation 2002;106(6):653-8.

7. Heitzer T, Schlinzig T, Krohn K,et al. Endothelial dysfunction, oxidative stress, and risk of cardiovascular events in patients with coronary artery disease. Circulation 2001;104(22):2673-8.

8. Perticone F, Ceravolo R, Pujia A, et al. Prognostic significance of endothelial dysfunction in hypertensive patients. Circulation 2001;104(2):191-6.

9. Suwaidi JA, Hamasaki S, Higano ST,et al. Long-term follow-up of patients with mild coronary artery disease and endothelial dysfunction. Circulation 2000;101(9):948-54.

10. Brunner H, Cockcroft JR, Deanfield J, et al. Endothelial function and dysfunction. Part II: association with cardiovascular risk factors and diseases. A statement by the Working Group on Endothelins and Endothelial Factors of the European Society of Hypertension. J Hypertens. 2005;23:233- 46 .

11. Suzuki T, Hirata K, Elkind MS, et al. Metabolic syndrome, endothelial dysfunction, and risk of cardiovascular events: the Northern Manhattan Study (NOMAS). Am Heart J. 2008;156(2):405-10.

12. Corretti MC, Anderson TJ, Benjamin EJ,et al. Guidelines for the ultrasound assessment of endothelial-dependent flow-mediated vasodilatation of the brachial artery: a report of the International Brachial Artery Reactivity Task Force. J Am Coll Cardiol 2002;39(2):257-65.

13. Gokce N, Keaney JF Jr, Hunter LM, et al. Predictive value of noninvasively determined endothelial dysfunction for long-term cardiovascular events in patients with peripheral vascular disease.J Am Coll Cardiol 2003;41(10):1769-75. 
14. Vita JA, Keaney JF Jr. Endothelial function: a barometer for cardiovascular risk? Circulation 2002;106(6):640-2.

15. Brunner H, Cockcroft JR, Deanfield J, et al. Endothelial function and dysfunction. Part II: association with cardiovascular risk factors and diseases. A statement by the Working Group on Endothelins and Endothelial Factors of the European Society of Hypertension. J Hypertens. 2005;23:233- 46

16. Benjamin EJ, Larson MG, Keyes MJ, et al. Clinical correlates and heritability of flow-mediated dilation in the community: the Framingham Heart Study. Circulation 2004;109:613-9.

17. Felmeden DC, Spencer CG, Blann AD, et al. Lowdensity lipoprotein subfractions and cardiovascular risk in hypertension: relationship to endothelial dysfunction and effects of treatment. Hypertension 2003;41: 528-33.

17. CockcroftJR, Chowienczyk PJ, Benjamin N, et al. Preserved endothelium-dependent vasodilatation in patients with essential hypertension. N. Engl. J Med. 1994;330:1036-40.

18. The Seventh Report of the Joint National Committee on Prevention, Detection, Evaluation, and Treatment of High Blood Pressure. JAMA 2003;289:2560-71.

19. Hashimoto M, Miyamoto Y, Matsuda Y, et al. New Methods to Evaluate Endothelial Function: Non-invasive Method of
Evaluating Endothelial Function in Humans. J Pharmacol Sci. 2003;93: $405-8$.

20. Celermajer DS, Sorensen KE, Gooch VM,et al. Noninvasive detection of endothelial dysfunction in children and adults at risk of atherosclerosis. Lancet. 1992; 340:1111-5.

22. Neunteufl T, Heher S, Katzenschlager R, et al. Late prognostic value of flow- mediated dilation in the brachial artery of patients with chest pain. Am J Cardiol. 2000;86:207-10.

23. Kuvin JT, Patel AR, Sliney KA, et al. Peripheral vascular endothelial function testing as a noninvasive indicator of coronary artery disease. J Am Coll Cardiol. 2001;38:18439.

24. Rossi R, Nuzzo A, Origliani G, et al. Prognostic role of flow-mediated dilation and cardiac risk factors in postmenopausal women. J. Am. Coll. Cardiol. 2008;51;9971002.

25. Chang HJ, Chung J, Choi SY, et al.Endothelial dysfunction in patients with exaggerated blood pressure response during treadmill test. Clin Cardiol. 2004;27(7):421-5.

26. Chang HJ, Chung JH, Choi BJ, et al. Endothelial dysfunction and alteration of nitric oxide/ cyclic GMP pathway in patients with exercise-inducedhypertension. Yonsei Med J. 2003;44(6):1014-2
Cite this article as: Mani Prasad Gautam, Samir Gautam, Usha Ghimire, et al. Endothelial dysfunction and the role of hypertension in Nepalese subjects with major coronary risk factors. Nepalese Heart Journal 2016;13(1):-13-18. 